\title{
Immunohistochemical Evaluation of Glucose Transporter Type 1 in Epithelial Dysplasia and Oral Squamous Cell Carcinoma
}

\author{
Karuza Maria Alves Pereira ${ }^{1}$, Sthefane Gomes Feitosa ${ }^{1}$, Ana Thayssa Tomaz \\ Lima $^{1}$, Ealber Carvalho Macedo Luna ${ }^{1}$, Roberta Barroso Cavalcante ${ }^{2}$, Kenio \\ Costa de Lima ${ }^{3}$, Filipe Nobre Chaves ${ }^{1}$, Fábio Wildson Gurgel Costa ${ }^{4 *}$
}

\begin{abstract}
Oral squamous cell carcinoma (OSCC) is the most common malignancy of the oral cavity and some of these have been documented in association or preceded by oral epithelial dysplasia (OED). Aggressive cancers with fast growth have demonstrated overexpression of some glucose transporters (GLUTs). Thus, the aim of this study was to analyze the immunohistochemical expression of the glucose transporter, GLUT-1, in OEDs and OSCCs, seeking to better elucidate the biological behavior of neoplasias. Fifteen cases were selected this research of both lesions. Five areas were analyzed from each case by counting the percentage of positive cells at $400 \mathrm{x}$ magnification. Immunoreactivity of GLUT-1 was observed in $100 \%$ of the samples ranging from $54.2 \%$ to $86.2 \%$ for the OSCC and $73.9 \%$ to $97.4 \%$ for the OED. Statistical test revealed that there was greater overexpression of GLUT-1 in OED than the OSCC ( $p=0.01)$. It is believed the high expression of GLUT-1 may reflect the involvement of GLUT-1 in early stages of oral carcinogenesis.
\end{abstract}

Keywords: Oral squamous cell carcinoma - oral epithelaial dysplaisa - GLUT - immunohistochemistry

Asian Pac J Cancer Prev, 17 (1), 147-151

\section{Introduction}

Among the malignant neoplasias of the oral cavity, the Oral Squamous Cell Carcinoma (OSCC) is the most common form, accounting for over $90 \%$ of the cases of these malignancies (Scully, 2005). Some OSCCs have also been documented in association with or preceded by a potentially malignant lesion, such as Oral Epithelial Dysplasia (OED). The prognosis of this carcinoma is still poor, particularly if it is discovered in the more advanced stages (Warnakulasuriya et al., 2008).

OED is a potentially malignant lesion, whose combination of cytological alterations and abnormal tissue architecture comprise the histopathological criteria for its diagnosis. This lesion demonstrates greater potential for undergoing malignant alteration to the squamous cell carcinoma, when compared with normal epithelial tissue (Warnakulasuriya et al., 2007).

Oral carcinogenesis is a complex process and various genes may be altered. These genetic alterations produce altered proteins, and an overexpression of these, with those of cell metabolism being those most recently pointed out (Demasi et al., 2010).

Tumor cells have the important property of substitution of oxidative phosphorylation by glucose as a predominant source of energy production (Demasi et al., 2010). The ATP (Adenosine Triphosphate) generated by glycolysis is the motor of mitosis, because it supplies energy to the nucleus (Moreno-Sanchez et al., 2009).

Glycolysis is not essential to the neoplasia, but constitutes a strategic opportunity favorable to survival and proliferation in circumstances of lack of nutrients and oxygen (Moreno-Sanchez et al., 2009).

Glucose is transported to within the cell by means of Glucose Transporters (GLUT), present in all types of cells. At present, various isoforms of GLUT have been described and the expression of these is cell specific, and subject to extracellular medium control. GLUT comprises a family with 12 members: GLUT 1-12 (Medina, Owen 2002; Macheda et al., 2005).

Functionally, the GLUTs regulate glucose transport between the extracellular medium and the intracellular compartment (Medina, Owen 2002; Macheda et al., 2005), carrying out the movement of glucose via the cellular membrane in tumor cells, facilitating the maintenance of glycolytic energy metabolism in cases of limited substrate.

An increase in the expression of certain GLUTs has been shown in various human cancers, such as carcinoma

${ }^{1}$ Department of Oral Pathology, School of Dentistry, Federal University of Ceará - Campus Sobral, Sobral, ${ }^{2}$ Department of Oral Pathology, Fortaleza University, Fortaleza, ${ }^{3}$ Department of Social and Preventive Odontology, School of Dentistry, Federal University of Rio Grande do Norte, Natal, ${ }^{4}$ Department of Clinical Dentistry, School of Dentistry, Federal University of Ceará, Fortaleza, Brazil*For correspondence: fwildson@yahoo.com.br 
of the esophagus, lung and prostrate, suggesting that the tumor cells use this metabolic pathway for their maintenance, and therefore proliferate so rapidly even in a medium at low oxygen tension. Moreover, the expression of these proteins has been correlated with the prognosis of tumors (Kunkel et al., 2003; Macheda et al., 2005; Moreno-Sanchez et al., 2007; Ayala et al., 2010; Ohba et al., 2010). The high expression of GLUT-1 is considered an important initial event for the development of cancers of the head and neck (Reisser et al., 1999).

The point of investigation of this research consists of verifying whether alterations in the glucose transport mechanism are strongly related to the malignant phenotype, and how these changes occur in initial stages of oral carcinogenesis, even in the OEDs, because it was verified that only the study of Reisser et al (1999), published in the American literature, compared the expression of GLUT-1 in OED and OSCC lesions.

Therefore, the aim of this study was to analyze the immunohistochemical expression of the glucose transporter, GLUT-1, in OEDs and OSCCs, seeking to better elucidate the biological behavior of malignant neoplasias.

\section{Materials and Methods}

This study was conducted by selecting 15 cases of OED, and 15 cases of OSCC in patients of the Outpatient Stomatology Clinic of the Federal University of Ceará - Sobral Campus, by means of incisional biopsy, in the period from August 2010 to December 2011.

This research was submitted to the Research Ethics Committee of the Ceará Dental Academy - Continual Education Center (ACO/CEC) for approval and obtained a favorable report in the year 2010.

The specimens with reference to the selected cases were fixed in $10 \%$ formol, and afterwards embedded in paraffin, submitted to $5 \mu \mathrm{m}$ (micrometers) thick cuts, stained with hematoxylin-eosin and mounted on glass slides for histopathological analysis.

After diagnostic confirmation, the specimens were once again submitted to $5 \mu \mathrm{m}$ thick cuts and mounted on glass slides previously prepared with an organosilanebased adhesive (3-aminopropyltriethoxysilane, Sigma Chemical Co®, St Louis, MO, USA).

The cuts were submitted to the anti-GLUT-1 antibody by the streptoavidin-biotin immunohistochemical technique (Labeled Streptavidin Biotin - LSAB). Briefly, it consisted of the following steps: the sections went through 2 baths in xylol, each lasting ten minutes. After this they were immersed in three passages of absolute alcohol, then washed in running water, and immediately after this, a passage in distilled water.

Antigen recovery was performed with citrate at $\mathrm{pH}$ 6.0 , for 30 minutes at $99 \mathrm{oC}$. After returning to ambient temperature, the sections were immersed in a $3 \%$ hydrogen peroxide blocking solution for 10 minutes. Next, they were incubated with the rabbit polyclonal antibody antiGLUT-1 (GTX15309, GeneTex®, San Antonio, TX, USA), for 60 minutes at Ambient Temperature (AT), at the dilution of 1:200, and afterwards washed with a phosphate buffered saline solution, PBS (phosphate buffered saline).

The samples were incubated with the secondary antibody LSAB Kit (DAKO®, Carpentaria, CA, USA) for 10 minutes at AT. Next, incubation was performed in a chromogen solution prepared with $\mathrm{DAB}$, for 10 minutes in a dark chamber (DAKO ${ }^{\circ}$, Carpentaria, CA, USA). Afterwards, the specimens were washed in running water and then in distilled water. Counter-staining was performed with hematoxylin, and afterwards the specimens were dehydrated in alcohol and diaphanized in xylol.

Lastly, they were mounted on glass slides, which were examined under a NIKON ${ }^{\circledR}$ Eclipse E200 optical microscope. As positive control, the erythrocytes were used, and parallel to the incubated sections, the negative control was performed, excluding the application of the primary antibody.

The parameter of positivity for the immunohistochemical marking of the antigen in all the specimens included in the sample consisted of the cells that exhibited brown staining in their cytoplasm, irrespective of the intensity of the immunomarking. Five fields were randomly selected, at 400x magnification, visualized through the abovementioned optical microscope, and photographed with a SONY® Cyber-shot digital camera, 7.2 mega pixels at maximum resolution.

Quantitative analysis of GLUT-1 protein expression was performed by counting the number, in absolute values, of immunomarked cells, by a single observer, at distinct time intervals, by means of the software program Image $\mathbf{J}$ (Image and Processing Analysis in Java - Rasband, W.S., ImageJ, National Institutes of Health, Bethesda, Maryland, USA, http://rsb.info.nih.gov/ij/, 1997-2004).

The results obtained were used to construct the database in an Excel spreadsheet, and data were afterwards transferred to the software program SPSS 17.0, Windows environment. For statistical analysis, the means, medians and the Mann-Whitney test were used, based on the levels of significance of $5 \%(\mathrm{p}<0.05)$.

\section{Results}

The sample consisted of 15 patients for each type of lesion, with 10 being of the male gender and 5 of the female for the carcinoma; and consisting of 6 men and 9 women for the dysplasia. The age-range was from 17 to 84 years, counting on younger patients in the OSCC, with the general mean of all ages being approximately within the range of 61 years for both lesions, which affected the various anatomic sites existent in the oral cavity. A higher percentage of patients of the male gender affected by OSCC and more advanced age were verified. As regards OED a higher percentage of patients of the female gender affected by this lesion and a younger age were verified.

GLUT-1 expression was positive in all the cases of OSCC and OED. GLUT-1 immunomarking was observed throughout the cell extension, with exception of the nucleus, however, intense marking was observed, particularly in the membrane of tumor cells (Figure 1) and dysplastic cells (Figures 2). The erythrocytes served as internal positive control (Figure 1). 
Immunohistochemical Evaluation of Glucose Transporter Type 1 in Epithelial Dysplasia and Oral Squamous Cell Carcinoma

Of the 15 cases selected for OSCC, the mean number of immunopositive cells ranged from approximately 54.2 $\%$ to $86.2 \%$, with the latter case being the highest number of cells marked (Figure 3 ). No significant association was observed between immunomarking of GLUT-1 with regard to the patients' age or gender. However, it was perceived that in $80 \%$ of the sample with immunoexpression of over $80 \%$ the anatomic site of the lesion was in the region of the base of the tongue and floor of the mouth.

Of the 15 cases selected for OED, the mean number of immunopositive cells ranged from approximately $73.9 \%$ to $97.4 \%$, with the latter case being the highest number of cells marked. Immunoexpression was shown particularly in the more inferior layers (Figure 4). No significant association was observed between immunomarking of GLUT-1 with regard to the patients' age and gender, and the anatomic site of the lesion.

For the OSCC specimens, evaluation of the total quantity of cells positive for GLUT-1 revealed a mean of $74.02 \%$; in turn, an OED revealed a mean of $85.44 \%$.

For statistical evaluation the software program SPSS 17.0 was used, in which the Mann-Whitney test was performed, and the following results obtained (Table 1).

When GLUT was compared between the lesions,

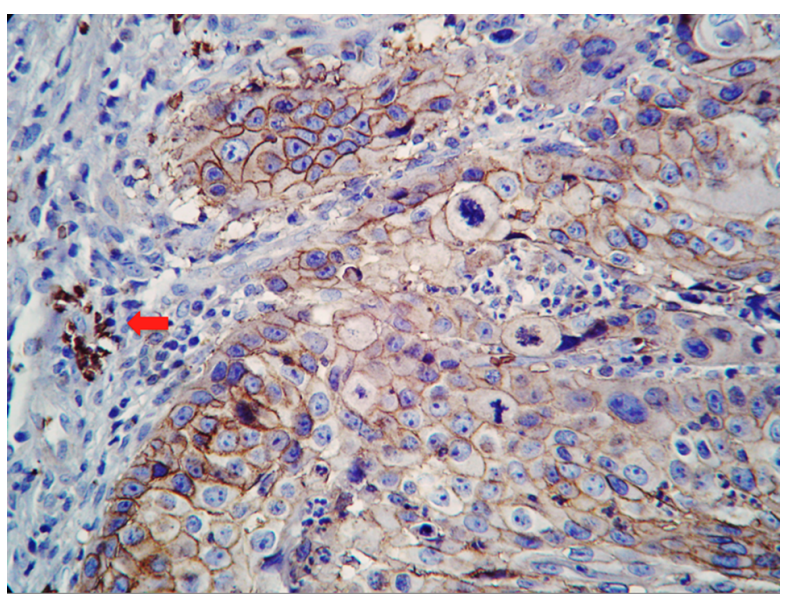

Figure 1. Photomicrograph Showing Intense Immunostaining of Membrane GLUT-1 in Cancer

Cells. The arrow indicates a positive internal control in erythrocytes (LSAB, $400 \times$ )

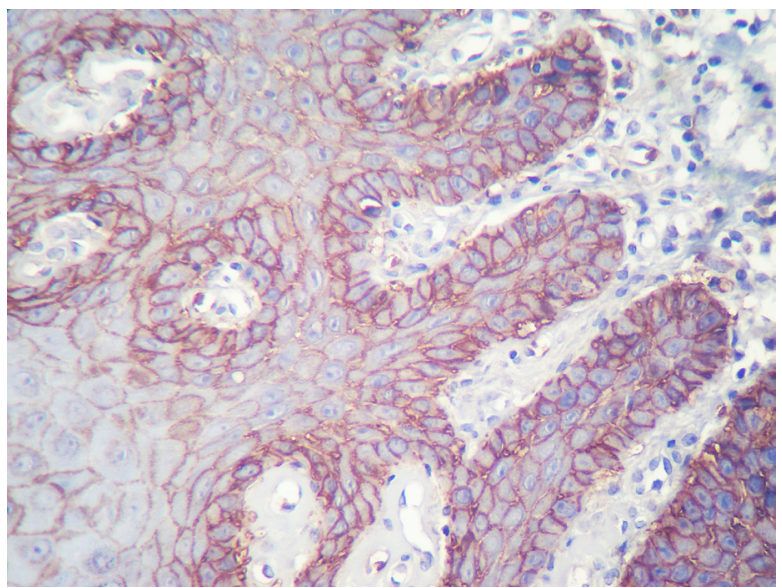

Figure 2. Photomicrograph Showing GLUT-1 Immunostaining in Cells Dysplastic Epithelium $($ LSAB, $400 \times)$

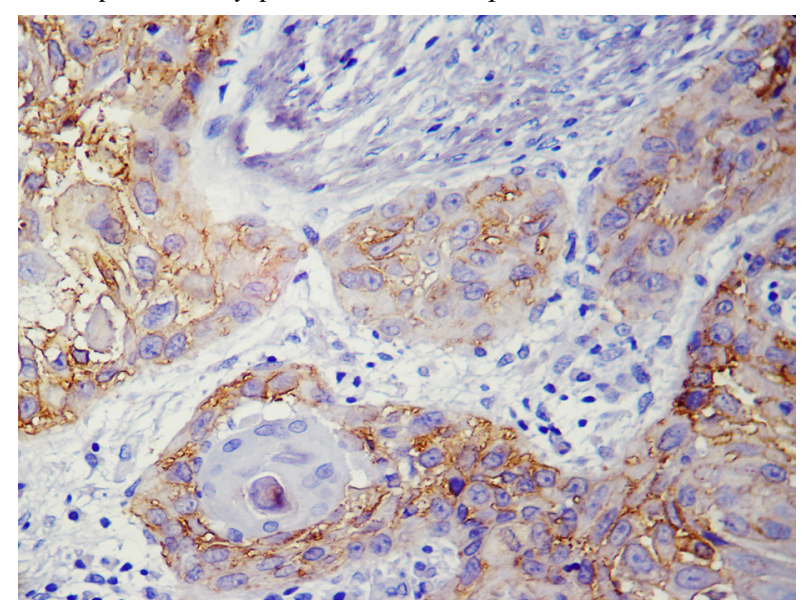

Figure 3. Oral Squamous Cell Carcinoma Photomicrograph Showing Varied Immunoexpression for GLUT-1 (LSAB, $400 \times$ )

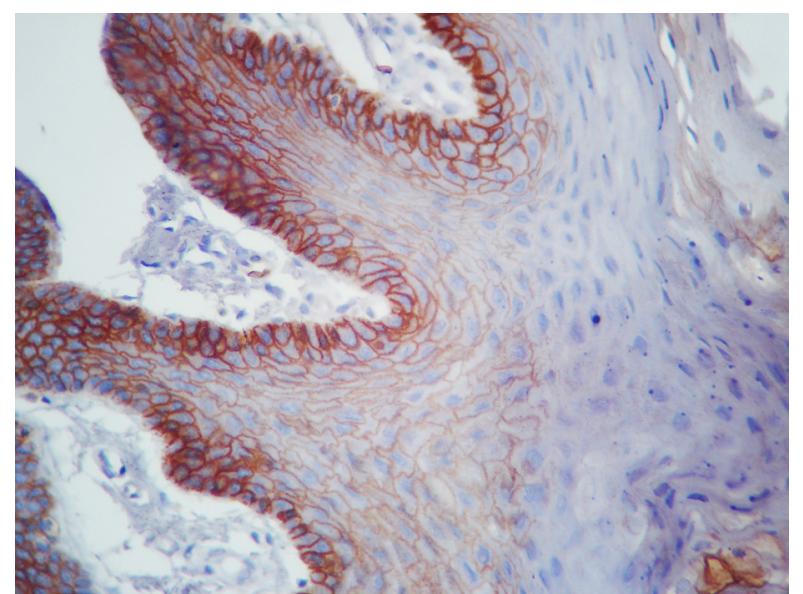

Figure 4. Oral Epithelial Dysplasia Photomicrograph Showing Intense GLUT-1 Immunoreactivity in Basal Layer Cells and Start in Spinous Layer (LSAB, 400 x).

Table 1. Number of Positive Cells for GLUT-1 (Oral Epithelial Dysplasia Versus Oral Squamous Cell Carcinoma)

\begin{tabular}{lccc}
\hline Oral lesions & Median & Q25 - Q75 & $\mathrm{P}^{*}$ \\
\hline Oral Epithelial Dysplasia & 85.05 & $78.33-95.61$ & 0.01 \\
Oral Squamous Cell Carcinoma & 75.71 & $65.13-82.36$ & \\
\hline
\end{tabular}

*Mann-Whitney test

significant difference was verified $(\mathrm{p}=0.01)$ between the immunomarking of the dysplasia and the carcinoma, with the former always having more marking.

\section{Discussion}

The present research showed evidence of the epidemiological pattern of the lesions (OED and OSCC), similarly to that which has been widely described in the literature as regards gender, age and location of lesions (Funk et al., 2002; Perussi et al., 2002; Reibel, 2003; Moreno-Sanchez et al., 2009).

The potentially malignant lesions are defined as those that have greater probability of developing into cancer. In spite of the great advancements in the understanding of tumor biology, it has still not been elucidated b some 
patients with these lesions develop cancer, while other do not (Warnakulasuriya et al., 2007).

Treatment planning and evaluation of the prognosis for squamous cell carcinoma, is mainly based on tumor staging by means of the TNM system (Sakashita et al., 2001; Oliveira, Ribeiro-Silva 2011). However, this classification provides limited information about the response to treatment as regards the biological aggressiveness of the tumor, because it becomes impossible to predict the prognosis with precision, even in patients with the same degree of staging (Heo et al., 2012).

Therefore, new molecular markers that seek to predict prognosis and tumor aggressiveness must be studied in an individualized manner, in the search for a treatment for OSCC, and for the lesions that may precede it.

The elevated expression of GLUT-1 is associated with the increase in cell metabolism, and this transporter is very expressive in a variety of human tumors and in normal tissues, such as erythrocytes, endothelial cells in cerebral vessels and fetal cells, in which there is increased use of glucose (Kato et al., 2007). Analyzing this protein may provide some subsidy for its prognosis, probably due to the increase in glycolytic metabolism of the more aggressive neoplastic cells (Ayala et al., 2010).

The alterations in GLUT-1 expression have been described as an early event during the development of not only squamous cell carcinomas, but in other carcinomas as well (Reisser et al., 1999). The overexpression of GLUT-1 has been observed in diverse types of malignancies apart from OSCC, such as in the esophagus, lung, stomach, colorectal region, breast and in the biliary vesicle (Haber et al., 1998; Mineta et al., 2002).

The immunohistochemical analysis in the present study demonstrated the marking of GLUT-1 in all the cases of OSCC. This result was in agreement with some studies that have obtained $100 \%$ of positive expression in all the analyzed cases of OSCC (Kato et al., 2007; Ohba et al., 2010; Heo et al., 2012); 90-95\% of positivity (Ayala et al., 2010) and differing from Eckert et al (2011), who obtained low expression of this protein. This fact suggests that GLUT is present in OSCC and probably indicates that the neoplastic cells may need this molecule for the development of the lesion.

The marking pattern strongly present in the cell membrane found in the present research was similar to that found in the studies of Haber et al (1998), Kato et al (2007), and Ayala et al (2010). The presence of GLUT1 in this location may reflect the main function of this protein, facilitating the maintenance of glycolytic energy metabolism of the cells, even those that are altered.

Furthermore, in the potentially malignant lesions, such as oral epithelial dysplasia's, a high expression of GLUT1 was observed, as was possible to verify in the present study. This is because this overexpression is observed under conditions in which the cells need glucose as a source of energy, such as during ischemia or hypoxia, which may occur as a result of an insufficient offer of oxygen through inefficient blood transportation (Reisser et al., 1999).

According to the high marking index observed in OED, it is believed that GLUT expression may be a determinant for the tumor phenotype, and therefore, it is suggested that this should be an indication of a malignant phenotype (Reisser et al., 1999; Mineta et al., 2002; Kunkel et al., 2003).

There are extremely few studies in the literature that compare the immunohistological evaluation of GLUT-1 in OSCC and OED, therefore there are no strong evidences that the dysplasia has more intense immunomarking than there is in the carcinoma. It has therefore not yet been elucidated whether the dysplasia has a more intense immunoexpression than the carcinoma has. In the present study, there was evidence of a greater immunoexpression of GLUT-1 in the dysplasia lesions than in the carcinoma. These findings may reflect the involvement of GLUT-1 in the initial stages of carcinogenesis, including in OEDs.

The justification for this finding is that the high levels of GLUT-1 expression have been correlated in diverse studies with the malignant transformation of cells and tumor malignancy phenotype (Reisser et al., 1999; Mineta et al., 2002; Kunkel et al., 2003; Moreno-Sanchez et a., 2009; Ohba et al., 2010).

Based on the results of this research, it was verified that there is greater GLUT-1 expression in the dysplastic lesions than in the carcinomas, differing from Reisser et al (1999), which was the only study published in the American literature, and it is believed that this fact must reflect the participation of GLUT in some determinant oncogenic mechanism for a tumor phenotype, and therefore, it is suggested that the overexpression of this protein is an indication of malignancy. Nevertheless, further studies are necessary, which prove this real association.

\section{Acknowledgements}

This research received the financial support of Grant \# BP1-0031-00088.01.00/10 from Fundação Cearense de Apoio ao Desenvolvimento Científico e Tecnológico (FUNCAP No. 02/2010).

\section{References}

Ayala FR, Rocha RM, Carvalho KC, et al (2010). GLUT1 and GLUT3 as potential prognostic markers for oral squamous cell carcinoma. Molecules, 15, 2374-87.

Demasi AP, Costa AF, Altemani A, et al (2010). Glucose transporter protein 1 expression in mucoepidermoid carcinoma of salivary gland: correlation with grade of malignancy. Int J Exp Pathol, 91, 107-13.

Funk GF, Karnell LH, Robinson RA, et al (2002). Presentation, treatment, and outcome of oral cavity cancer: a National Cancer Data Base report. Head Neck, 24, 165-80.

Haber RS, Rathan A, Weiser KR, et al (1998). GLUT1 glucose transporter expression in colorectal carcinoma: a marker for poor prognosis. Cancer, $\mathbf{8 3}, 34-40$.

Heo K, Kim YH, Sung HJ, et al (2012). Hypoxia-induced upregulation of apelin is associated with a poor prognosis in oral squamous cell carcinoma patients. Oral Oncol, 48, 500-6.

Kato Y, Tsuta K, Seki K, et al (2007). Immunohistochemical detection of GLUT-1 can discriminate between reactive mesothelium and malignant mesothelioma. Mod Pathol, 20, 215-20. 
Immunohistochemical Evaluation of Glucose Transporter Type 1 in Epithelial Dysplasia and Oral Squamous Cell Carcinoma

Kunkel M, Reichert TE, Benz P, et al (2003). Overexpression of Glut-1 and increased glucose metabolism in tumors are associated with a poor prognosis in patients with oral squamous cell carcinoma. Cancer, 97, 1015-24.

Macheda ML, Rogers S, Best JD (2005). Molecular and cellular regulation of glucose transporter (GLUT) proteins in cancer. J Cell Physiol, 202, 654-62.

Medina RA, Owen GI (2002). Glucose transporters: expression, regulation and cancer. Biol Res, 35, 9-26.

Mineta H, Miura K, Takebayashi S, et al (2002). Prognostic value of glucose transporter 1 expression in patients with hypopharyngeal carcinoma. Anticancer Res, 22, 3489-94.

Moreno-Sanchez R, Rodriguez-Enriquez S, Marin-Hernandez A, Saavedra E (2007). Energy metabolism in tumor cells. Febs J, 274, 1393-418.

Moreno-Sanchez R, Rodriguez-Enriquez S, Saavedra E, MarinHernandez A, Gallardo-Perez JC (2009). The bioenergetics of cancer: is glycolysis the main ATP supplier in all tumor cells? Biofactors, 35, 209-25.

Ohba S, Fujii H, Ito S, et al (2010). Overexpression of GLUT-1 in the invasion front is associated with depth of oral squamous cell carcinoma and prognosis. J Oral Pathol Med, 39, 74-8.

Oliveira LR, Ribeiro-Silva A (2011). Prognostic significance of immunohistochemical biomarkers in oral squamous cell carcinoma. Int J Oral Maxillofac Surg, 40, 298-307.

Perussi MR, Denardin OV, Fava AS, Rapoport A (2002). [Squamous cell carcinoma of the mouth in the elderly in Sao Paulo]. Rev Assoc Med Bras, 48, 341-4.

Reibel J (2003). Tobacco and oral diseases. Update on the evidence, with recommendations. Med Princ Pract, 1, 22-32.

Reisser C, Eichhorn K, Herold-Mende C, Born AI, Bannasch P (1999). Expression of facilitative glucose transport proteins during development of squamous cell carcinomas of the head and neck. Int J Cancer, 80, 194-8.

Sakashita M, Aoyama N, Minami R, et al (2001). Glut1 expression in T1 and T2 stage colorectal carcinomas: its relationship to clinicopathological features. Eur J Cancer, 37, 204-9.

Scully C (2005). Oral cancer; the evidence for sexual transmission. Br Dent J, 199, 203-7.

Warnakulasuriya S, Johnson NW, van der Waal I (2007). Nomenclature and classification of potentially malignant disorders of the oral mucosa. J Oral Pathol Med, 36, 575-80.

Warnakulasuriya S, Reibel J, Bouquot J, Dabelsteen E (2008). Oral epithelial dysplasia classification systems: predictive value, utility, weaknesses and scope for improvement. J Oral Pathol Med, 37, 127-33. 